\title{
Coulomb explosion as a probe to understand the mechanism of electron stripping from ions interacting with crystalline solids
}

\author{
David Martín y Marero, ${ }^{1,2,3, *}$ Nuria Gordillo, ${ }^{1}$ and Raquel González-Arrabal ${ }^{4}$ \\ ${ }^{1}$ Centro de Microanálisis de Materiales, Universidad Autónoma de Madrid, 28049 Madrid, Spain \\ ${ }^{2}$ Parque Científico de Madrid, Campus de Cantoblanco, 28049 Madrid, Spain \\ ${ }^{3}$ Departamento de Física Aplicada, Universidad Autónoma de Madrid, 28049 Madrid, Spain \\ ${ }^{4}$ Instituto de Microelectrónica de Madrid, CNM-CSIC, 28760 Tres Cantos, Spain
}

(Received 7 January 2009; published 30 April 2009)

\begin{abstract}
When an ion impinges on a solid, it rapidly undergoes a process in which its electrons are stripped away provided the velocity of the orbiting electrons is smaller than the projectile speed. Electron stripping determines any posterior behavior of the ions in the solid, and it is assumed that it takes place on the surface of the solid, but no information is available on the details of the process. Here we show, using the Coulomb explosion of $\mathrm{C}_{2}^{+}$ions moving in $\mathrm{Si}$ as a tool, that electron stripping takes place in an orderly manner and that the number of electrons stripped, before charge equilibration, depends on a characteristic length. We also propose a relation capable of quantifying this dependence. We foresee these results as a starting point to a more general understanding of ion-solid interaction, with important consequences on ion beam analysis and modification techniques, and special significance in silicon technology.
\end{abstract}

DOI: 10.1103/PhysRevB.79.155449

PACS number(s): 61.85.+p, 34.50.Bw, 34.50.Fa, 34.70.+e

\section{INTRODUCTION}

\section{A. Historical and objectives}

Ion beam analysis (IBA) and ion beam modification (IBM) are well-established techniques widely employed in many different fields of knowledge, ranging from fundamental physics to fine arts, with special impact on surface and materials science. These techniques developed in the early 1960s, as the Van de Graaff accelerators used for nuclear physics research were not able to produce the higher energies required in this field and new uses were devised for them. IBA techniques are based in the detection and analysis of the products emitted from the different processes induced when an energetic ion strikes on a target, whereas IBM uses the capability of a foreign ion to change the native properties of a system, either due to its energy or due to its chemical difference. Now, we are in the presence of mature techniques which nevertheless continue to find new fields of application, while the lack of knowledge on some fundamental aspects persists. One of these aspects is the first moment of the interaction of a beam of ions with a solid, in particular the stripping of the ion electrons by the target in the first few atomic layers. This is not a new subject, and as far as 1948, Bohr ${ }^{1}$ proposed the criterion which explained under which conditions the projectile would lose electrons to the target, basically, that the electron's orbital velocity should be lower than the velocity of the ion. It is assumed that stripping takes place on the surface of the solid, but no information exists on the details of the process or the role of the target's structure on the process. It is largely agreed that while in the target, the ions undergo a rapid electron loss, which is followed by a subsequent charge exchange, ${ }^{2}$ but the issue of where and how this rapid electron loss occurs, before any charge exchange toward equilibrium starts, remains an open question. Indeed, in the literature this fact is obviated and is always mentioned as happening "after traversing a few atomic layers" or "within a very short time $(\ll 1 \mathrm{fs}),{ }^{, 3-7}$ without further clarification. Electron stripping is an effect that will determine the ion's charges in deeper layers and largely any posterior behavior, including the stopping force or energy loss per unit length, $S=-d E / d x .^{8}$ This relation between electron stripping and $S$ has been made clear in previous studies, where charge state and energy loss have been quantified from the same physical model, including the demonstration that an atomic ion loses more energy than its neutral counterpart, ${ }^{9}$ or the explicit inclusion of charge state populations to calculate the electronic stopping power from first-principles calculations. ${ }^{10}$ Further works have provided general expressions for the ion's mean energy loss and its fluctuation, straggling, in the presence of charge exchange, for slow ${ }^{11}$ and swift ${ }^{12}$ ions, encompassing in the later case, the determination of the transient behavior of charge exchange straggling and the skewness of the energy-loss profile.

On the subject of charge exchange itself, several successful attempts have been made to measure the average charges of ions as they traverse a solid, either experimentally at the exit of the solid ${ }^{3,4}$ or in the case of computer simulations ${ }^{13}$ and theory, ${ }^{14}$ with a prior assignment of an initial charge state of the ion followed by a calculation of the evolving charge state while in the solid. Consequently, the charge is measured after charge equilibration has taken place to some extent, and the information contained in the first few layers, or how the initial state was acquired, either is lost in the experimental case or in the case of simulation and theory has not been the subject under study.

In this work, we determine the number of electrons stripped from molecular ions during their interaction with the first atomic layers of a crystalline solid. We use as a probe the Coulomb explosion occurring between the constituents of the molecular ions after the removal of the electrons. By performing these measurements in channeling condition along different crystallographic directions, experimentally and with Monte Carlo computer simulations, we are able to provide light on fundamental aspects of the effect of the 
target's atomic distribution on the stripping. Furthermore, of relevant significance is the selection of silicon as the target sample, not only from a theoretical point of view but because of its main role in any modern technological development, where the use of ion beam techniques with energies verifying Bohr criteria, is routinely deployed for its modification and further analysis. Another successful example of the use of the imprint that the electron's stripping and subsequent Coulombian repulsion have, in this case for a practical application, is the direct imaging of the structure and dynamics of flexible and excited molecules. $5,6,15,16$

\section{B. Definition of terms}

An incident ion beam can penetrate into a crystalline solid target in two different configurations, the so-called random and channeling. Random occurs when incoming ions cover erratically distributed paths within the target solid, and it may occur in every solid crystalline or not. Channeling takes place when the incoming ions are constrained to move between atomic rows or planes along the crystallographic axis or planes in a crystalline solid. In this case, the ions follow well-defined paths or trajectories, undergoing successive small-angle collisions at each crystal plane and experiencing an oscillatory steering effect. ${ }^{17,18}$ Experimentally, channeling can be detected using the Rutherford backscattering (RBS) technique, appearing as a reduction in yield of the number of backscattered ions from target atoms when compared with random incidence or incidence on an amorphous solid target. ${ }^{19}$ An exhaustive review on channeling for nonspecialists can be found in Ref. 19.

The rich array of phenomena taking place when atomic ions moves under channeling conditions-such as dragging forces on the projectiles as the target electrons are distributed behind as a trailing wake, ${ }^{1,20}$ with major fundamental consequences on the motion of ions in dense media, ${ }^{21-23}$ transverse heating or increase in the transversal momentum due to inelastic scattering with target electrons, ${ }^{24,25}$ transverse cooling, and heating due to repeated capture and loss of electrons ${ }^{26}$ - is augmented by new effects when the target is bombarded with molecular instead of monoatomic ions. Upon entering a solid, a molecular ion is also stripped of its bonding electrons in the first monolayers, and thus, the Hamiltonian which describes the system experiences an abrupt switch from that of a bound molecular ion to a net Coulombian repulsion between the constituent atoms. ${ }^{27,28}$ In this repulsion process, part of the internal potential energy is then transformed into transverse kinetic energy, giving rise to an increase in the number of close encounters with atomic rows. This effect, termed as Coulomb explosion, is observed in channeling configuration as an enhancement of the backscattering yield dechanneling when compared to atomic ions. This and other effects, as the alignment of the clusters under the effect of the electrons plasma wake, ${ }^{29}$ vicinage, or the coherent dynamic response by the target electrons in the presence of correlated ions ${ }^{3}$ or the alteration of the chargestate equilibrium in the presence of molecular ions, ${ }^{30}$ have induced a vast amount of research on molecular clusters, ${ }^{31}$ from theoretical and experimental approaches, not only be- cause of the intrinsic interest of correlated motion of particles in a crystalline lattice and in particular their effect on the stopping power of ions but also due to their practical applications: for inertial confinement fusion in large scale facilities; ${ }^{32,33}$ as mentioned above, for direct imaging the structure of molecules, which in contrast to traditional techniques allows the study of excited states and photochemical dynamics, ${ }^{5,6,15,16}$ as well as the modification of materials at a nanometer scale. .,34,35 $^{2}$

In spite of all this research carried out up to now on the Coulomb explosion, to our knowledge, the Coulomb explosion has not been used to understand such a basic aspect of the ion-solid interaction as the process of the electron's stripping of the impinging ions. However, here we face the study of the removal of the electrons from a different perspective, considering the role of the target's atomic position in the process, showing how the spatial periodicity of the target's atoms influences the rate at which the stripping of the electrons occurs.

\section{EXPERIMENTAL}

\section{A. Layout and the basics of the measurements}

The experiments were performed at the Standard Beamline of the $5 \mathrm{MV}$ tandetron accelerator of the Centro de $\mathrm{Mi}$ croanálisis de Materiales (CMAM) of the Universidad Autónoma de Madrid. ${ }^{36}$ A cesium sputter source, (HVEE 860), a graphite cathode, and a $\mathrm{N}_{2}$ stripper gas were used to generate atomic, $\mathrm{C}^{+}$, and molecular, $\mathrm{C}_{2}^{+}$, ions, while an analyzing magnet was used to select the ions species. The ions were accelerated to $1.847 \mathrm{MeV} /$ atom and directed toward a [100] silicon single crystal, which was chemically etched with HF acid to remove the native oxide surface layer. The RBS channeling $(\mathrm{RBS} / \mathrm{C})$ measurements were performed along the $\langle 100\rangle,\langle 111\rangle$, and $\langle 211\rangle$ directions. The scattering geometry was optimized to take into account the limited resolution by placing a silicon surface-barrier detector $(\Delta E \approx 45 \mathrm{keV})$ at a scattering angle of $120^{\circ}$ for the first case and at $80^{\circ}$ for the rest, achieving kinematic factors of 0.25 and 0.47 , respectively. ${ }^{37}$ Measurements at different ion energies of the position of the high-energy edge allowed the precise determination of $\delta E$, i.e., the energy per channel and $E_{0}$, the lower limit of energy that the detector was able to measure. To compare measurements done with $\mathrm{C}^{+}$and $\mathrm{C}_{2}^{+}$and be able to detect if any Coulomb explosion takes place, care was taken to have ions reaching the sample with the same energy per atomic unit and that the number of units is also the same. For the first condition it was of paramount importance the voltage stability of the accelerator, ${ }^{38}$ whereas several steps were taken to ensure the second: in order to eliminate charges other than the scattered ions at the detector, an electron suppressor ring biased at $-180 \mathrm{~V}$ was placed at the entrance of the sample chamber and a bias voltage of $+160 \mathrm{~V}$ was applied to the sample holder; spots in the crystal were irradiated homogeneously and with the same fluency by limiting the beam size with slits instead of focusing; and finally, the spectra were corrected for the detector dead time by normalizing to the counts of a pulse generator. The spectra were 

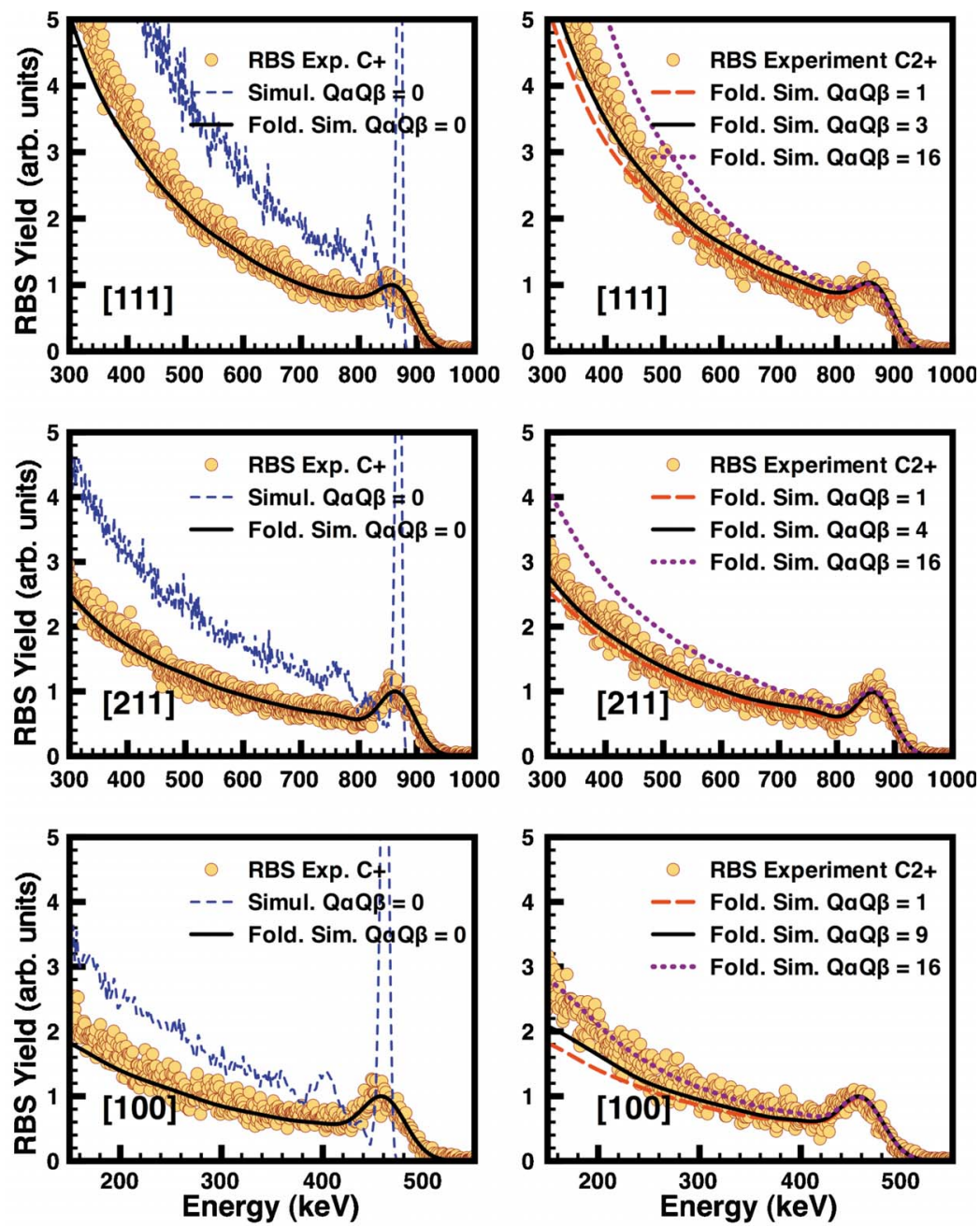

FIG. 1. (Color online) Experimental and simulated backscattering yields. Left: RBS measured spectrum (open circles) with $\mathrm{C}^{+}$beams on silicon for three channeling directions. Also shown the RBS simulated spectra before (dashed line) and after convolution (fold. sim.) with the experimental resolution (solid line). From these results it is clear that the simulations describe adequately the experimental yield obtained from the atomic beams. Right: RBS measured spectrum (open circles) with $\mathrm{C}_{2}^{+}$beams compared with simulations, showing the effect that different values of $Q_{\alpha} Q_{\beta}$, the product of the individual charges of each atom that constitutes the $\mathrm{C}_{2}$ molecule, have on the spectra. The different energy scale for $\langle 100\rangle$ is due to the smaller kinematic factor. For purposes of display the data has been normalized to the surface peak maximum. corrected for the damage produced by successive irradiations by calculating the average yield increase per irradiation and subtracting this quantity times the irradiation number.

Basically, the experimental procedure consisted in a sequential set of irradiations of the same virgin spot with $\mathrm{C}^{+}$, followed by $\mathrm{C}_{2}^{+}$and two more irradiations with $\mathrm{C}^{+}$beams. This sequential irradiation procedure allowed for the radiation-induced crystal damage correction and for a deconvolution between the enhancement in the backscattered yield related to the damage contribution and to that due to the Coulomb explosion effect. A detailed description of the experimental procedure, including damage correction, is given elsewhere. ${ }^{39}$

In general, two windows may be considered in each of the channeling spectra, one close to the sample surface, which would be highly influenced by phenomena taking place at the crystal surface and the other one, the bulk region, which would be the appropriate one to properly study the Coulomb explosion resulting from the stripping of the electrons in the surface region. Figure 1 shows the collected RBS data. The bulk region corresponds to energies below $\sim 800 \mathrm{keV}$ for the $\langle 111\rangle$ and $\langle 211\rangle$ directions and below $\sim 420 \mathrm{keV}$ for the $\langle 100\rangle$ direction. The surface region is indicated by the peak above those energies. From now on we are going to focus on the bulk region. After damage correction, an enhancement in the backscattering yield is clearly visible for the three chan- neling directions as displayed in Fig. 2, where the integrated intensities of the RBS spectra shown in Fig. 1 are plotted as a function of the irradiation step. The second irradiation step corresponds to $\mathrm{C}_{2}^{+}$, whereas the rest to $\mathrm{C}^{+}$beams. On the top row, the integration of the pulsed normalized data is presented, and the bottom row shows the same data after damage correction. These integrated intensities were calculated in the bulk region considering a number of channels such that $E \in[300,800] \mathrm{keV}$ along $\langle 111\rangle$ and $\langle 211\rangle$ and $E$ $\in[200,400] \mathrm{keV}$ along $\langle 100\rangle$. Integrated intensities calculated for other energy intervals, always inside the bulk, produced negligible relative differences. Measurements in random configuration for both molecular and atomic ions provided the same backscattering yield, confirming that for equivalent current integrator counts the spectra due to atomic and molecular beams were the same. This result, equal yield for random configuration, also confirms that other effects such as vicinage can be neglected within the experimental uncertainties. ${ }^{31}$ In channeling configuration, and as expected if any Coulomb explosion has taken place, the yield due to atomic ions was lower since the atomic ions can travel a longer path along the channel, whereas for molecular ions experiencing a Coulomb explosion, the transverse kinetic energy increases the close encounters with the target's atoms, producing a larger yield. 

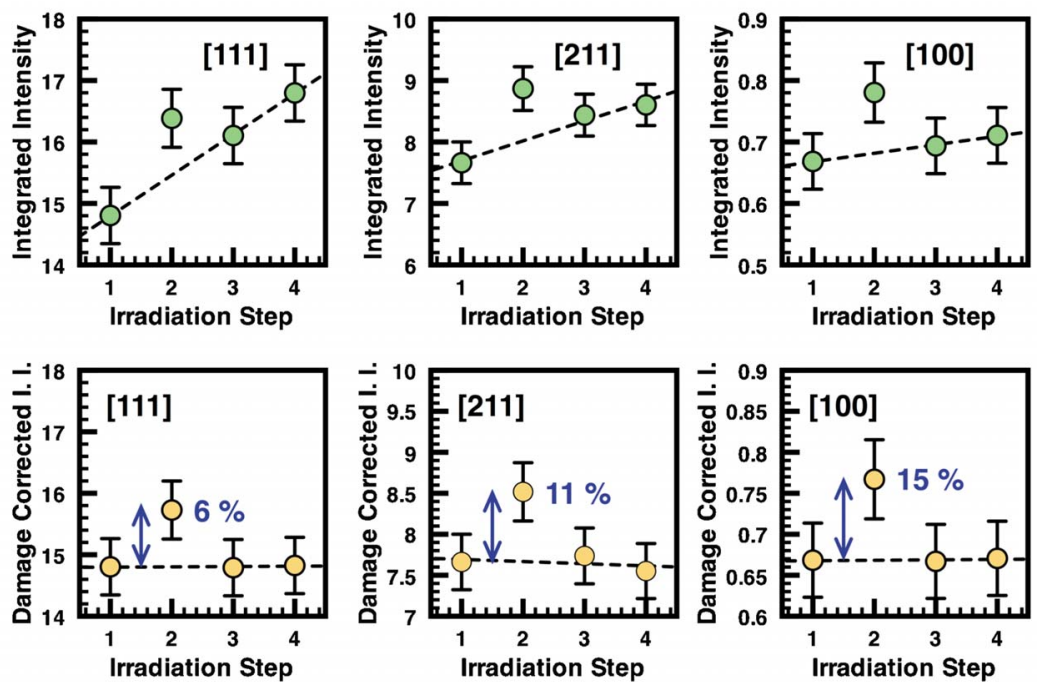

FIG. 2. (Color online) The signature of the Coulomb explosion. Integrated intensities of the Rutherford backscattering spectra measured in channeling configuration (RBS/C) before (top) and after (bottom) damage correction. The second irradiation step corresponds to $\mathrm{C}_{2}^{+}$beams. Dashed lines are a fit to the atomic irradiations, showing the linear character of the radiation-induced damage. The damage correction was carefully done by calculating the average yield increase per irradiation step of an atomic beam and subtracting this quantity times the irradiation number. The increase in yield from atomic to molecular beam is the signature of the Coulomb explosion and is the quantity to compare with the simulations.

\section{B. Validation of the Monte Carlo simulations}

In order to obtain information about the charge of each atom in the molecular ion before charge equilibration takes place, the equations of motion of the atomic and molecular ions while in the crystal lattice, were solved using Monte Carlo computer simulation via a customization of the program UPIC, ${ }^{40}$ where the mutual Coulomb interaction of the ions that constitutes the molecule, is now taken into account. ${ }^{41}$ To simulate the ion flux behavior in channeling and in random configuration for atomic ions, the Coulomb interaction was set to zero. Thus $Q_{\alpha} Q_{\beta}=0$, where $Q_{\kappa}$ is the charge of atom $\kappa$ of a molecular ion. For the molecular ions simulations, all the possible values of ionization of the carbon atom were considered, as will be detailed below.

The program represents a crystal structure as a series of primitive parallelepiped cells with a single atom on each one, and its thermal displacement is taken into account as a random distribution which follows the Debye model. The interaction potential that an ion experiences due to a crystal atom is based on the Thomas-Fermi potential, $V(r)$ $=\varphi(r / \mu) K Z e / r$, where $K=1 / 4 \pi \epsilon_{0}$ is the electrostatic constant, $Z$ is the crystal atom atomic number, $e$ is the charge of the electron, $r$ is the distance to the atom, and $\varphi(r / \mu)$ is a screening function to the Coulomb potential, with $\mu$ as the screening length. The program uses the Molière's approximation $\varphi(r / \mu)=\Sigma_{i} p_{i} \exp \left(-q_{i} r / \mu\right)$, with $p_{i}$ $=\{0.35,0.55,0.10\}$ and $q_{i}=\{0.3,1.2,6.0\},{ }^{42}$ as in practice it describes scattering often better than a numerical solution to the Thomas-Fermi equation for atoms. The local electrondensity approach is followed, so the terms contributing to the energy loss on each primitive cell are proportional to the electron density on the ion's trajectory. Two terms were considered: $\Delta E_{v}$, due to the cession of energy to the valence electrons, which are assumed to be uniformly distributed over the crystal, and $\Delta E(b)$, resulting from the excitations of the atomic cores for an impact parameter $b$. The reference stopping cross sections ${ }^{43}$ are ensured by a single factor normalization and the screening length $\mu$ was optimized to correct the limitations of the local electron-density approach. Multiple scattering of ions by electrons were taken into ac- count, resulting in a velocity dispersion after traversing each cell. ${ }^{1}$ The initial position of a carbon ion at the crystal surface is chosen at random and for the molecular case, the time and place of arrival of the other carbon atom is determined by the orientation of the molecule, which has equal probabilities in all directions. In order to improve the statistics of the computer experiments and reduce spurious effects, five simulations with different seeds of the random number generator were performed for each studied value of $Q_{\alpha} Q_{\beta}$ and then averaged. This method also allowed the calculation of the uncertainties. ${ }^{44}$ Orientation of the simulated crystals were set in the same three channeling directions of the experimental study and also in random. The random configuration was selected to inspect differences in yield between atomic and molecular beams. As in the experimental case, no difference was observed in random configuration, which constitutes a test of the validity of the potential used by the simulation program.

The simulated data are shown for the atomic case in Fig. 1 left column, where the peak around $460 \mathrm{keV}$ for irradiation along $\langle 100\rangle$ and at $900 \mathrm{keV}$ for $\langle 111\rangle$ and $\langle 211\rangle$ is the first peak of the normalized nuclear encounter probability $P_{\mathrm{NE}}$ or surface peak. ${ }^{44}$ The peak around $440 \mathrm{keV}$ for $\langle 100\rangle, 840 \mathrm{keV}$ for $\langle 111\rangle$, and $820 \mathrm{keV}$ for $\langle 211\rangle$, corresponds to the second peak of $P_{\mathrm{NE}}$ or the first peak below the surface, due to those particles which are not channeled, but their movement is still governed by the crystal structure and move across the channel center between opposite walls, until they interact with the nearest-neighbor rows. The third peak is clearly evident at around 400,820 , and $760 \mathrm{keV}$, respectively, due to interactions with outer nearest-neighbor rows and hence a greater height than the second peak. ${ }^{19,45}$ The observation of these peaks and their mentioned characteristics also adds to the reliability of the simulation procedure.

The accumulated backscattering yield of the simulated data $Y_{0}$ does not take into account the finite experimental angular resolution. Therefore, simulated and experimental data cannot be directly compared; but to contrast them in a proper manner, the former had to be folded with the experimental resolution. The final simulated intensity to compare with the experimental data is then given by $I_{\text {Sim }}(E)=A_{0}\left[Y_{0}\right.$ 
$\otimes R(E)]$, where $A_{0}$ is a scale factor equal to the ratio between the experimental and simulated yields in random orientation, so the spectra can be compared directly; $R(E)$ is a normalized Gaussian fitted to the experimental resolution, with a full width at half maximum (FWHM) of $72 \pm 1 \mathrm{keV}$ at $80^{\circ}$ for $\langle 111\rangle$ and $\langle 211\rangle$, and $50 \pm 1 \mathrm{keV}$ at $120^{\circ}$ for $\langle 100\rangle$, whereas $\otimes$ stands for the convolution. After convolution, the peaks are smeared out almost completely except for the peak at zero depth or surface peak, as it happens with the experimental data, and both sets of data, experimental and simulated, can be compared directly.

It is important to mention that the same scale factor, $A_{0}$, was successfully used to match the simulated and experimental spectra of atomic ions impinging under channeling orientation. This is a very relevant fact because it confirms that the model and specially the potential employed by the program and which describes the interaction between the impinging ions and the target nuclei can be used for different orientations. Therefore, it constitutes a stringent assessment of the quality of the simulations. In particular, it is a proof that the stopping cross sections, which are very different for channeling and random configurations, naturally arise as the simulated crystal is rotated. The correct fitting between simulated and experimental data in the case of the atomic beams, $Q_{\alpha} Q_{\beta}=0$, also implies that the model used, which does not take into account any charge exchange process, is valid in the bulk region selected for the study, as proven also in Ref. 41. Evidently, the same value of $A_{0}$ was also used for all the molecular ion cases, $Q_{\alpha} Q_{\beta} \neq 0$, but in this case, $Q_{\alpha} Q_{\beta}$ was varied independently for all the possible combinations of the ionization states of the $\mathrm{C}_{2}^{+}$molecule: from $Q_{\alpha} Q_{\beta}=1$ to $Q_{\alpha} Q_{\beta}=36$. The resulting simulated spectra are then compared to the second experimental irradiation made with molecular beams. Therefore, any differences in simulated yield between $Q_{\alpha} Q_{\beta} \neq 0$ (molecular beam) and $Q_{\alpha} Q_{\beta}=0$ (atomic beam) are exclusively due to the existence of charges producing the Coulomb explosion effect. Experimental RBS spectra taken with a molecular-beam and simulated RBS spectra for three different $Q_{\alpha} Q_{\beta}$ values are shown, after damage corrections, in Fig. 1 (right column). This figure evidences that the simulated combination of charges which better fits the experimental data depends on the channeling direction. In contrast, the case of single ionization of each atom of the molecule, $Q_{\alpha} Q_{\beta}=1$, and the case of full ionization of the $L$ shell, $Q_{\alpha} Q_{\beta}=16$, gives rise to lower and higher yields, respectively, as also shown in Fig. 1. Hence, this combination of charges, $Q_{\alpha} Q_{\beta}$, constitutes the only parameter used to compare simulation and experiment and obtain the underlying microscopic information, as described in detail in Sec. III.

\section{DISCUSSION}

\section{A. Determination of charges and phenomenological description}

The conclusions achieved in Sec. II legitimate us to use the Coulomb explosion as a tool to study the electron stripping process that suffer molecular ions in the solid before any further charge exchange and equilibration takes place.
The influence of the electron stripping in the succeeding Coulomb explosion is evaluated by calculating the increment in the integrated intensity associated to the bulk region in the $\mathrm{RBS} / \mathrm{C}$ spectra when $\mathrm{C}^{+}$is replaced by $\mathrm{C}_{2}^{+}$. Figure 3 shows these calculated increments for all the possible $Q_{\alpha} Q_{\beta}$ values in units of squared electron charge (filled circles), together with the observed experimental increment, $\Delta I$, (dashed horizontal line). From these data the average charge state, $Q_{\alpha} Q_{\beta}$, can be inferred as the intersection value between the measured and the simulated data. Again, a clear dependence of the average charge state on the channeling directions is observed, being $Q_{\alpha} Q_{\beta}$ equal to 2.8 for $\langle 111\rangle$, 4.6 for $\langle 211\rangle$, and 9 for $\langle 100\rangle$. A possible physical magnitude to explain this dependence is the channels' width, since the narrower the channel, the greater the interaction of the ions with the atomic rows configuring the channels. This width is routinely expressed as the area in the plane normal to a row, $\left(N d_{\text {row }}\right)^{-1}$, where $N$ is the density of atoms in the lattice and $d_{\text {row }}$ is the distance between them along the row. ${ }^{44}$ Note that this magnitude must not be confused with the standard spacing between lattice planes $(h k l)$ in a crystal, $d_{h k l}$. Both distances are tabulated in Table I for comparison. Alternatively, the dependence of the average charge on the geometrical area, $A_{\mathrm{Geom}}$, can be considered. As can be noticed from Table I, no evident correlation exists between $Q_{\alpha} Q_{\beta}$ and $\left(N d_{\text {row }}\right)^{-1}$, neither with $A_{\mathrm{Geom}}$, indicating that these magnitudes seem not to be responsible for the observed dependence on the channeling direction. A different approach is to shift the attention in the direction parallel to the rows instead of the direction in the planes perpendicular to them. To this purpose, a spatial period is calculated for the periodic structures depicted in Fig. 3 by taking into account the periodicity of the crystal and the atoms that shape the channels form. Let $T_{h k l}$ be the length traveled by an ion between two equivalent atoms along a channel. For axes $\langle 100\rangle$ and $\langle 211\rangle, T_{h k l}$ coincides with $d_{\text {row }}$, whereas for axis $\langle 111\rangle, T_{h k l}$ is the sum of the two possible values of $d_{\text {row }}$ (see Table I). By comparing the $T_{h k l}$ values with those obtained for $Q_{\alpha} Q_{\beta}$ an inverse relation becomes clear. Defining the average charge of each of the atoms of the molecule moving along $[h k l]$ as $Q_{h k l}=\sqrt{Q_{\alpha} Q_{\beta}}$, obviating $Q_{211}$ for now, and taking into account the experimental uncertainties so $Q_{\alpha} Q_{\beta}=3$ for $\langle 111\rangle$ and $Q_{\alpha} Q_{\beta}=9$ for $\langle 100\rangle$, we have $Q_{100} T_{100}=Q_{111} T_{111} \simeq 3 a \approx 16.3 e \AA$, where $a$ is the lattice parameter. In fact, the calculated values obtained for $Q_{100} T_{100}$ and $Q_{111} T_{111}$ are consistent with this relation within uncertainties, as shown in Table I. Therefore, the inverse relation between $T_{h k l}$ and $Q_{\alpha} Q_{\beta}$ is purely geometrical and depends on the atomic positions along the crystallographic channels. The importance of the atomic positions becomes excruciating when $\langle 211\rangle$ is studied since $Q_{211} T_{211}$ is around $87 \%$ of $3 a$, a difference which cannot be attributed to the experimental error. Nevertheless, this deviation can be explained by symmetry, inasmuch as the atoms shaping $\langle 100\rangle$ and $\langle 111\rangle$ are all at the same distance from their respective channel center, whereas for $\langle 211\rangle$, we have two sets of atoms at different distances: those labeled with asterisks in Fig. 3 are farther. This interpretation is supported by very recent $a b$ initio simulations using time-dependent density-functional theory, which confirms that the interaction of an ion moving on a crystalline solid is extremely local and is much larger 

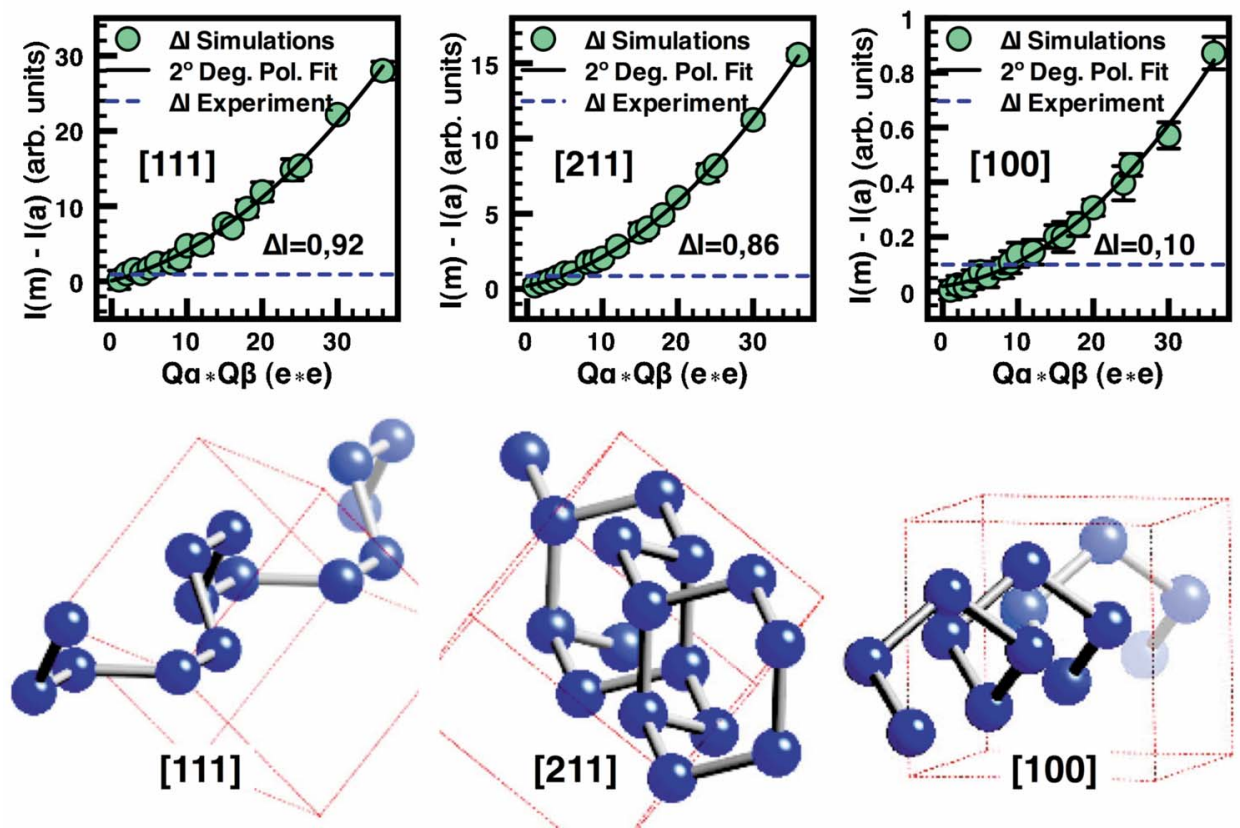

FIG. 3. (Color online) Matching of the computer simulated yield increment to the experimental values for each channel and a representation of the channels. Top: difference in integrated intensities between $\mathrm{C}_{2}^{+}, I(m)$, and $\mathrm{C}^{+}, I(a)$, in the bulk region of the simulations and their intersection with the experimental values depicted in Fig. 2. From this intersection, the ion's charges are inferred. Bottom: image of the [111], [211], and [100] channels. For $\langle 111\rangle$ the fractional coordinates of the atoms that form the channel are of type: $(0,0,0),(1 / 4,1 / 4,1 / 4),(1 / 2$, $0,1 / 2),(3 / 4,1 / 4,3 / 4),(1 / 2,1 / 2,1),(3 / 4,3 / 4,5 / 4)$, and $(1,1,1)$; for $\langle 100\rangle:(0,1 / 2,1 / 2),(1 / 4,3 / 4,3 / 4),(1 / 2,1 / 2,1),(3 / 4,1 / 4,3 / 4)$, and $(1$, $1 / 2,1 / 2)$; and for $\langle 211\rangle$ : $(1 / 4,1 / 4,1 / 4),(0,1 / 2,1 / 2),(1 / 2,0,1 / 2)^{*},(1 / 4,3 / 4,3 / 4)^{*},(3 / 4,1 / 4,3 / 4),(1 / 2,1 / 2,1),(1,1 / 2,1 / 2)^{*}$, $(3 / 4,3 / 4,5 / 4)^{*},(5 / 4,3 / 4,3 / 4)$, and $(1,1,1)$. Those positions labeled with asterisks are farther from the channel center.

with the atoms closest to the trajectory than with any other. ${ }^{46}$ Notwithstanding further theoretical developments, the following phenomenological relation is proposed:

$$
\frac{Q_{h k l}}{Q_{h^{\prime} k^{\prime} l^{\prime}}}=\frac{T_{h^{\prime} k^{\prime} l^{\prime}}}{T_{h k l}}
$$

applicable to channels along $\langle h k l\rangle$ and $\left\langle h^{\prime} k^{\prime} l^{\prime}\right\rangle$ if their atoms lie at the same distance from the channel center.

\section{B. Microscopical picture}

We are now in position to provide a physical picture. When a molecular ion impinges on a target, a binding electron can be lost to the first atom it encounters which is nearer than the characteristic distance for electron loss. In the case of molecular ions penetrating into a crystalline solid in channeling configuration, the molecular ion has a specific orientation with respect to the crystal lattice, which makes it continue its path along the crystallographic axis without encountering any other equivalent atom, until it traverses a length $T_{h k l}$, where it may lose another electron. The orientation of the molecule could be altered by the wake forces of the leading particles; but this effect is negligible in the spatial period $T_{h k l}$ since any reorientation of the molecule occurs after traveling very long distances: $\sim 10^{2}-10^{3} \AA$ for alignments of $0.1^{\circ} \cdot{ }^{29}$ Ergo, after only three or four spatial periods, while the ions are still in what is considered the surface, the stripping of the ions' electrons that determines the succeeding Coulomb explosion has taken place, and since the

TABLE I. Magnitudes obtained after combined experimental and Monte Carlo analysis. $d_{h k l}$ is the spacing between planes $[h k l] ; d_{\text {row }}$ is the distance between atoms in a row; $\left(N d_{\text {row }}\right)^{-1}$ is the channel's width, with $N$, the density of atoms in the lattice; $A_{\mathrm{Geom}}$ is the geometrical area normal to the channel; $T_{h k l}$ is the spatial period along channel $[h k l] ; Q_{\alpha} Q_{\beta}$ is the product of charges matching the experiment; and $Q_{h k l}$ denotes $\sqrt{Q_{\alpha} Q_{\beta}}$ along channel $[h k l]$.

\begin{tabular}{lccccccc}
\hline \hline$[h k l]$ & $\begin{array}{c}d_{h k l} \\
(\AA)\end{array}$ & $\begin{array}{c}d_{\text {row }} \\
(\AA)\end{array}$ & $\begin{array}{c}\left(N d_{\text {row }}\right)^{-1} \\
\left(\AA^{2} / \text { at. }\right)\end{array}$ & $\begin{array}{c}A_{\mathrm{Geom}} \\
\left(\AA^{2}\right)\end{array}$ & $\begin{array}{c}T_{h k l} \\
(\AA)\end{array}$ & $\begin{array}{c}Q_{\alpha} Q_{\beta} \\
\left(e^{2}\right)\end{array}$ & $\begin{array}{c}Q_{h k l} T_{h k l} \\
(e \AA)\end{array}$ \\
\hline$[111]$ & 3.14 & $4.70^{\mathrm{a}}$ & 4.26 & 2.14 & 9.41 & $2.8 \pm 0.4$ & $15.8 \pm 1.1$ \\
{$[211]$} & 2.22 & 6.65 & 3.01 & 5.68 & 6.65 & $4.6 \pm 0.7$ & $14.2 \pm 1.1$ \\
{$[100]$} & 5.43 & 5.43 & 3.69 & 3.71 & 5.43 & $9 \pm 2$ & $16.6 \pm 1.8$ \\
\hline \hline
\end{tabular}

${ }^{\mathrm{a}}$ For $\langle 111\rangle$ there are two possible values of $d_{\text {row }}: 2.35$ and $7.06 \AA$, so their average is used (Ref. 19). 
strength of the repulsive interaction during these initial states is at its peak, it determines any subsequent behavior. This means that although charge exchange may take place afterwards, by then, the particles that were composing the molecule are far apart and behaving without any mutual correlation so the imprint that the electron stripping left is permanent. Further support for this general picture is the time taken for the electron stripping to occur, which is in agreement with the accepted time for removing the binding electrons: within a very short time $(\ll 1 \mathrm{fs})$. In our case, 1.847 MeV/atom $\mathrm{C}_{2}^{+}$on $\mathrm{Si}$, it takes around $0.1 \mathrm{fs}$ to travel $T_{100}, 0.12$ fs for $T_{211}$, and 0.2 fs for $T_{111}$.

\section{SUMMARY AND CONCLUSIONS}

To summarize, using the Coulomb explosion as a tool, we have brought to light the details of a long-standing problem: the first moments of the interaction of ions with matter or how the stripping of the electrons occurs. This stripping determines any posterior behavior since it is the starting point for charge exchange and equilibration, as well as the most important determination of the stopping force. We have iden- tified $T_{h k l}$ as the physical parameter behind the observations and propose a phenomenological relation for the calculation of the resulting charges. As an application of Eq. (1) and based on $T_{110} \simeq 3.84 \AA$, we can predict $Q_{110} \simeq 3 \sqrt{2} e$, i.e., a lost of at least all the electrons of the $L$ shell in the case of carbon ions. More precise measurements are foreseen using new instrumentation now under commissioning, ${ }^{47}$ as well as experiments with different projectiles and targets to corroborate the universality of our findings.

\section{ACKNOWLEDGMENTS}

This work was financed by the Ministerio de Ciencia y Tecnología (MCYT) and Ministerio de Educación y Ciencia (MEC) of Spain under Grant No. MAT2005-03011, "Ramón y Cajal" (D.M.M.), "Juan de la Cierva" (R.G.-A.), and "Personal Técnico de Apoyo" (N.G.) Programmes; and by the Madrid Government and Universidad Autónoma de Madrid under Grant No. CCG07-UAM/MAT-1882/07. We thank V. A Khodyrev and D. O. Boerma for very helpful discussions and access to the simulation program UPIC.
*Corresponding author; david.martinymarero@uam.es

${ }^{1}$ N. Bohr, Mat. Fys. Medd. K. Dan. Vidensk. Selsk. 18, 1 (1948).

${ }^{2}$ D. Zajfman, G. Both, E. P. Kanter, and Z. Vager, Phys. Rev. A 41, 2482 (1990).

${ }^{3}$ W. Brandt, A. Ratkowski, and R. H. Ritchie, Phys. Rev. Lett. 33, 1325 (1974).

${ }^{4}$ A. Breskin, A. Faibis, G. Goldring, M. Hass, R. Kaim, Z. Vager, and N. Zwang, Phys. Rev. Lett. 42, 369 (1979).

${ }^{5}$ I. Plesser, Z. Vager, and R. Naaman, Phys. Rev. Lett. 56, 1559 (1986).

${ }^{6}$ Z. Vager, R. Naaman, and E. P. Kanter, Science 244, 426 (1989).

${ }^{7}$ Y.-N. Wang, H.-T. Qiu, and Z. L. Misković, Phys. Rev. Lett. 85, 1448 (2000).

${ }^{8}$ W. Brandt and M. Kitagawa, Phys. Rev. B 25, 5631 (1982).

${ }^{9}$ A. Närmann, R. Monreal, P. M. Echenique, F. Flores, W. Heiland, and S. Schubert, Phys. Rev. Lett. 64, 1601 (1990).

${ }^{10}$ A. Arnau, M. Peñalba, P. M. Echenique, F. Flores, and R. H. Ritchie, Phys. Rev. Lett. 65, 1024 (1990).

${ }^{11}$ A. Närmann, W. Heiland, R. Monreal, F. Flores, and P. M. Echenique, Phys. Rev. B 44, 2003 (1991).

${ }^{12}$ A. Närmann and P. Sigmund, Phys. Rev. A 49, 4709 (1994).

${ }^{13}$ F. Grüner, F. Bell, W. Assmann, and M. Schubert, Phys. Rev. Lett. 93, 213201 (2004).

${ }^{14}$ V. S. Malyshevsky, Phys. Rev. B 72, 094109 (2005).

${ }^{15}$ Z. Vager, E. P. Kanter, G. Both, P. J. Cooney, A. Faibis, W. Koenig, B. J. Zabransky, and D. Zajfman, Phys. Rev. Lett. 57, 2793 (1986).

${ }^{16}$ Ch. Ellert, H. Stapelfeldt, E. Constant, H. Sakai, J. Wright, D. M. Rayner, and P. B. Corkum, Philos. Trans. R. Soc. London, Ser. A 356, 329 (1998).

${ }^{17}$ M. T. Robinson and O. S. Oen, Phys. Rev. 132, 2385 (1963).

${ }^{18}$ G. R. Piercy, F. Brown, J. A. Davies, and M. McCargo, Phys. Rev. Lett. 10, 399 (1963).
${ }^{19}$ D. S. Gemmell, Rev. Mod. Phys. 46, 129 (1974).

${ }^{20}$ J. Neufeld and R. H. Ritchie, Phys. Rev. 98, 1632 (1955).

${ }^{21}$ V. N. Neelavathi, R. H. Ritchie, and W. Brandt, Phys. Rev. Lett. 33, 302 (1974).

${ }^{22}$ P. M. Echenique, R. H. Ritchie, and W. Brandt, Phys. Rev. B 20, 2567 (1979).

${ }^{23}$ A. Mazarro, P. M. Echenique, and R. H. Ritchie, Phys. Rev. B 27, 4117 (1983).

${ }^{24}$ R. S. Nelson and M. W. Thompson, Philos. Mag. 8, 1677 (1963).

${ }^{25}$ J. Lindhard, Mat. Fys. Medd. K. Dan. Vidensk. Selsk. 34, 1 (1965).

${ }^{26}$ W. Assmann, H. Huber, S. A. Karamian, F. Grüner, H. D Mieskes, J. U. Andersen, M. Posselt, and B. Schmidt, Phys. Rev. Lett. 83, 1759 (1999).

${ }^{27}$ J. C. Poizat and J. Remillieux, Phys. Lett. A 34, 53 (1971).

${ }^{28}$ T. A. Tombrello and J. M. Caywood, Phys. Rev. B 8, 3065 (1973).

${ }^{29}$ D. S. Gemmell, J. Remillieux, J. C. Poizat, M. J. Gaillard, R. E. Holland, and Z. Vager, Phys. Rev. Lett. 34, 1420 (1975).

${ }^{30}$ B. T. Meggitt, K. G. Harrison, and M. W. Lucas, J. Phys. B 6, L362 (1973).

${ }^{31}$ N. R. Arista, Nucl. Instrum. Methods Phys. Res. B 164-165, 108 (2000) for a review of the current knowledge.

${ }^{32}$ R. J. Beuhler, G. Friedlander, and L. Friedman, Phys. Rev. Lett. 68, 2108 (1992).

${ }^{33}$ J. Zweiback, R. A. Smith, T. E. Cowan, G. Hays, K. B. Wharton, V. P. Yanovsky, and T. Ditmire, Phys. Rev. Lett. 84, 2634 (2000).

${ }^{34}$ H.-P. Cheng and J. D. Gillaspy, Phys. Rev. B 55, 2628 (1997).

${ }^{35}$ H. Dammak, A. Dunlop, D. Lesueur, A. Brunelle, S. DellaNegra, and Y. Le Beyec, Phys. Rev. Lett. 74, 1135 (1995).

${ }^{36}$ D. J. W. Mous, A. Gottdang, R. G. Haitsma, G. García López, A. 
Climent-Font, F. Agulló-López, and D. O. Boerma, in Application of Accelerators in Research and Industry, edited by J. L. Duggan and I. L. Morgan, AIP Conf. Proc. No. 680 (AIP, New York, 2003), pp. 999-1002.

${ }^{37}$ The kinematic factor is given by $K_{S}=\left\{\left[M_{1} \cos \theta+\left(M_{2}^{2}\right.\right.\right.$ $\left.\left.\left.-M_{1}^{2} \sin ^{2} \theta\right)^{1 / 2}\right] /\left(M_{1}+M_{2}\right)\right\}^{2}$, where $\theta$ is the scattering angle, $M_{1}$ and $M_{2}$ are, respectively, the projectile and target atom masses, with $M_{1}<M_{2}$.

${ }^{38}$ A. Climent-Font, F. Pászti, G. García, M. T. Fernández-Jiménez, and F. Agulló, Nucl. Instrum. Methods Phys. Res. B 219-220, 400 (2004).

${ }^{39}$ R. González-Arrabal, V. A. Khodyrev, N. Gordillo, G. García, and D. O. Boerma, Nucl. Instrum. Methods Phys. Res. B 249, 65 (2006).
${ }^{40}$ V. A. Khodyrev, V. Y. Chumanov, G. P. Pokhil, and K. K. Bourdelle, Nucl. Instrum. Methods Phys. Res. B 94, 523 (1994).

${ }^{41}$ V. A. Khodyrev, V. S. Kulikauskas, and C. Yang, Nucl. Instrum. Methods Phys. Res. B 195, 259 (2002).

${ }^{42}$ G. Molière, Z. Naturforsch. A 2, 133 (1947).

${ }^{43}$ J. F. Ziegler, J. P. Biersack, and W. Littmark, The Stopping and Range of Ions in Matter (Pergamon Press, Oxford, 1985).

${ }^{44}$ J. H. Barrett, Phys. Rev. B 3, 1527 (1971).

${ }^{45}$ J. H. Barrett, Phys. Rev. Lett. 31, 1542 (1973).

${ }^{46}$ J. M. Pruneda, D. Sánchez-Portal, A. Arnau, J. I. Juaristi, and E. Artacho, Phys. Rev. Lett. 99, 235501 (2007).

${ }^{47}$ D. Martín y Marero, O. Enguita, J. G. Zubiri, A. Rodríguez, J. Narros, and D. O. Boerma, Nucl. Instrum. Methods Phys. Res. B 249, 253 (2006). 